\title{
Anomalous atmospheric temperature perturbations over seismically active regions of Europe according to satellite measurements
}

\author{
Leonid Sverdlik ${ }^{1, *}$, Sanjar Imashev ${ }^{1}$, and Tatiana Yamskikh ${ }^{2}$ \\ ${ }^{1}$ Research Station of the Russian Academy of Sciences in Bishkek, Kyrgyzstan \\ ${ }^{2}$ Siberian Federal University, Institute of Space and Information Technology, Russian Federation
}

\begin{abstract}
An algorithm for defining temperature anomalies and the results of diagnostic studies, based on a retrospective analysis of time series of satellite data in troposphere and stratosphere over the epicenters of strong earthquakes with magnitude $M>6.0$, occurring in seismically active Mediterranean region of Europe are presented. It was found out that in all cases anomalous temperature disturbances were registered 1-10 days before the main seismic event.
\end{abstract}

\section{Introduction}

In recent years, there have been a number of studies, addressing the results of retrospective satellite remote sensing data analysis, which showed correlation between seismic activity and anomalous temperature variations in the upper troposphere during the catastrophic earthquake in Japan in spring 2011 [1], as well as on the eve of strong seismic events on the territory of Russia [2], in the northern Tien-Shan chains [3] and other seismically active regions of Asia from equator to mid-latitudes, both over land and ocean surfaces (Fig. 1) [4]. Despite the general positive statistics of temperature anomalies that preceded strong earthquakes, it was still impossible to state that these tropospheric effects are inherent to all major seismic events. Thus, there arose an obvious necessity for additional research, the main purpose of which, besides the development of methods for defining anomalous temperature perturbations in the tropopause, would be to enlarge the statistics of strong earthquakes. At the same time, we proceeded from the fact that analysis of connection between the dynamics of atmospheric and lithospheric processes, regardless of the method used, is based on the assumption that variations of parameters caused by seismic activity differ significantly from background fluctuations that occur during the periods when there were not recorded strong earthquakes, and, consequently, on allocation of areas connected with seismic activity in which a typical anomalous behavior in temporal data sequences using appropriate integral indicators was registered [5].

This paper describes the improved algorithm for defining short-period anomalous changes in the spatial-temporal distribution of temperature, and presents the results that were

\footnotetext{
*Corresponding author: 1. sverdlik@mail.ru
} 
obtained with the proposed algorithm application to strong earthquakes with magnitude $\mathrm{M}>$ 6.0 in the Mediterranean region of Europe.

\section{Seismic and satellite data}

Within the frameworks of this study, we analyzed results of atmospheric temperature satellite measurements on the eve of 8 strong earthquakes with magnitudes in the range 6.1 to 7.1 , which were recorded in 2011-2016 in Turkey, Italy and Greece (Table 1 and Fig. 1). Seismic event data were obtained from interactive map service IRIS Earthquake Browser (IEB) (http://ds.iris.edu/).

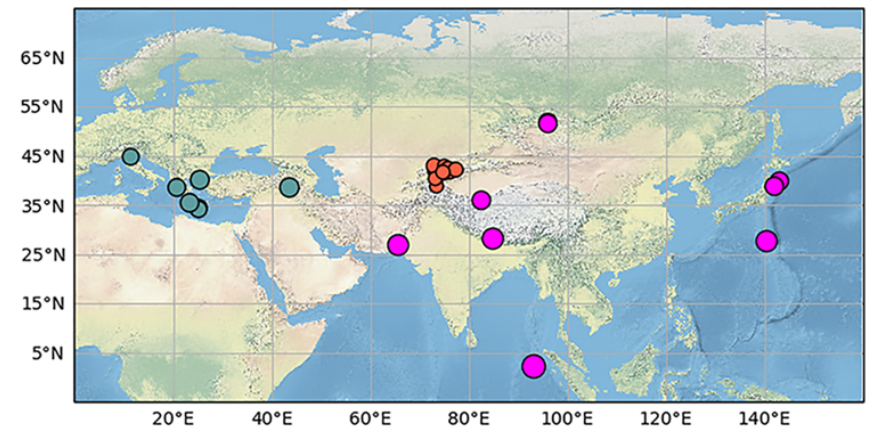

Fig. 1. The location of earthquakes epicenters.

Table 1. The features of seismic events under study $(M>6.0)$.

\begin{tabular}{|c|c|c|c|c|c|l|}
\hline № & Date & Time & Latitude N & Longitude E & M & \multicolumn{1}{|c|}{ Region } \\
\hline EQ01 & 23.10 .2011 & $10: 41: 22$ & 38.72 & 43.51 & 7.1 & Turkey \\
\hline EQ02 & 20.05 .2012 & $02: 03: 53$ & 44.87 & 11.22 & 6.1 & Italy \\
\hline EQ03 & 15.06 .2013 & $16: 11: 02$ & 34.40 & 25.02 & 6.3 & Greece, Crete \\
\hline EQ04 & 16.06 .2013 & $21: 39: 05$ & 34.35 & 25.16 & 6.1 & Greece, Crete \\
\hline EQ05 & 12.10 .2013 & $13: 11: 52$ & 35.49 & 23.26 & 6.8 & Greece, Crete \\
\hline EQ06 & 24.05 .2014 & $09: 25: 03$ & 40.30 & 25.45 & 6.9 & Aegean Sea \\
\hline EQ07 & 17.11 .2015 & $07: 10: 07$ & 38.67 & 20.60 & 6.5 & Greece \\
\hline EQ08 & 30.10 .2016 & $06: 40: 19$ & 42.85 & 13.09 & 6.6 & Italy \\
\hline
\end{tabular}

To analyze spatial and temporal variation of atmospheric temperature we used satellite measurements (AIRS / Aqua and MERRA-3D) for 10 pressure levels $\left(p_{k}\right)$ varying from 500 to $70 \mathrm{hPa}$ (http://giovanni.gsfc.nasa.gov/) covering the altitude from the upper troposphere to the lower stratosphere. The vertical profiles of the temperature $T(p)$ were reconstructed over each earthquake epicenter $\left( \pm 1^{\circ}\right)$ for time periods of at least 2-3 months before and after each of the EQ01-EQ08 events. The temporal discretization of initial temperature data $T(t)$ was $\Delta t=3 \mathrm{~h}$.

\section{Algorithm for processing the results of satellite measurements of atmospheric temperature}

The algorithm for calculating the integral indicators of anomalous variations of temperature time series includes 6 main stages and is based on the use of a traditional approach, which 
consists in decomposing data into long and short-term components, with subsequent filtering and removing smooth trends.

\subsection{Preprocessing of satellite data}

At the first stage we formed continuous time series with different time resolutions $(\Delta t=3 \mathrm{~h}$, $12 \mathrm{~h}$ and $24 \mathrm{~h}$ ) for each of the seismic events, containing temperature values at all altitude levels of atmosphere from 500 to $70 \mathrm{hPa}$. The duration of observation series $T(t)$ was 4-6 months. The height of the tropopause and cases of double tropopause were also determined on the basis of WMO criteria from temperature profiles $T(p)$ [6].

\subsection{Spectral analysis of atmospheric temperature time series}

Due to unsteady and non-linear nature of the factors influencing atmospheric temperature changes, large-scale (low-frequency) components of time series were detected using spectral analysis based on continuous wavelet transform. Using wavelet spectrograms we could reveal a hidden periodicity (quasi-permanent components), which must be eliminated along with low-frequency seasonal trend.

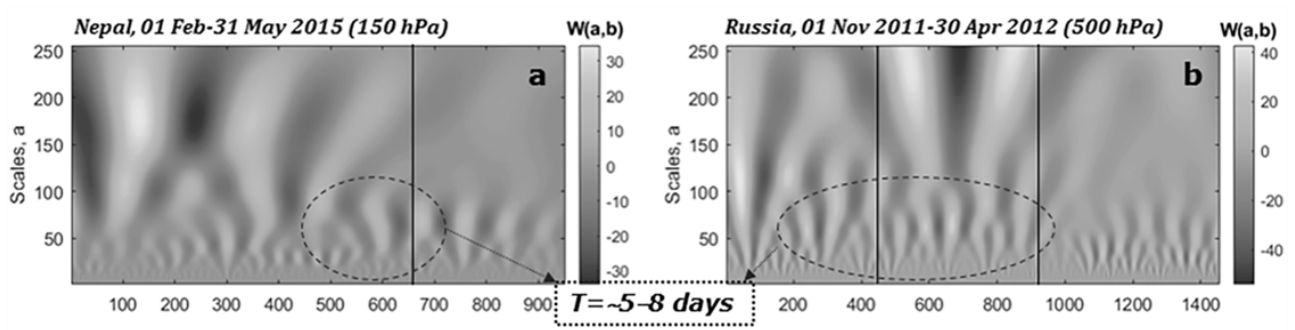

Fig. 2. Wavelet spectrograms of temperature time series $(\Delta t=3 \mathrm{~h})$ during periods of seismic activity in Nepal (150 hPa) (a) and Russia (500 hPa) (b). Vertical lines show the moments of strong earthquakes.

\subsection{Allocation of short-term temperature variations}

Processing of temperature time series is based on the analysis of quasi periodic oscillations with periods $\sim 5-8$ days. The validity of this approach is shown by wavelet spectrograms, clearly demonstrating variations of a similar time scale before strong earthquakes (Fig. 2). To allocate short-term variations we applied the nonlinear threshold-filtering method based on discrete wavelet transform [7], which has several advantages in comparison to classical methods, for example using moving average or higher-order polynomials.

\subsection{Selection of height ranges}

A fact-based selection of altitude ranges for carrying out calculations is very important for temperature data analysis. This procedure consists of determining the most informative pressure levels that are appropriate for specific locations, taking into account the features in the dynamics of thermal structure. For this, in each case we calculated correlation coefficients between temperature variations for each pair of levels (Fig. 3).

Temperature variations are non-uniform and differ significantly in separate atmospheric layers: the highest positive correlation is observed in the free troposphere, it decreases with increasing altitude to almost zero at the border of the troposphere and stratosphere and 
changes sign in the zone of transition to positive gradients $(\gamma>\Delta T / \Delta p)$. Square correlation matrix, which dimension corresponds to the number of the layers considered $p_{k}$, is a convenient way to represent the results visually. Based on such a matrix representation of the initial temperature data, it is possible to select reasonably the zones separated by the tropopause with the most intense antiphase oscillations (Fig. 3).
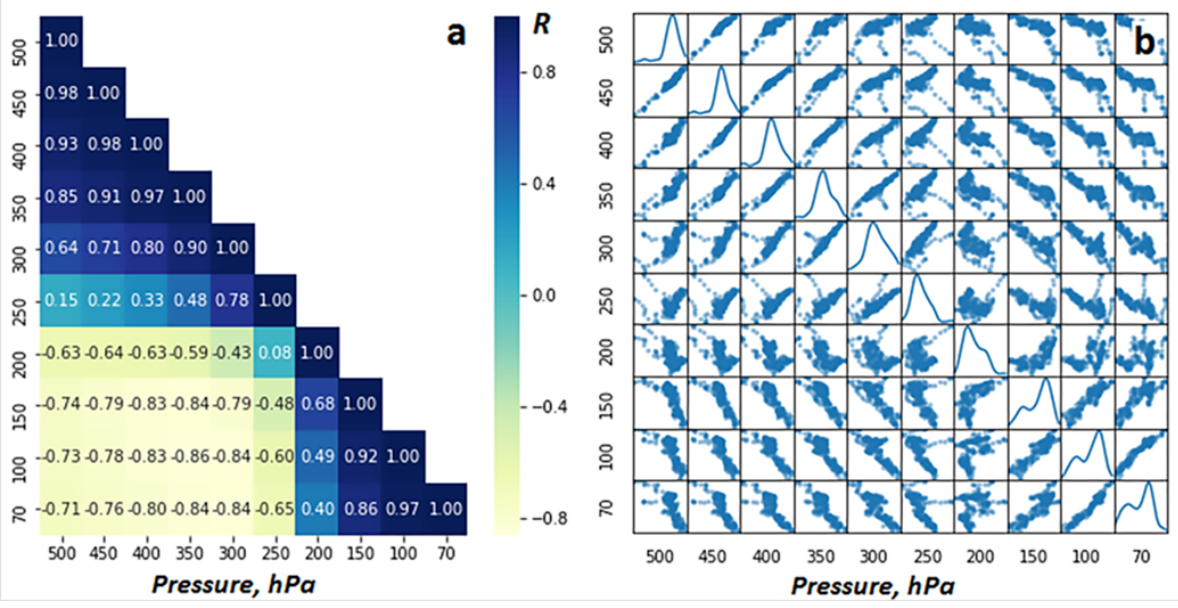

Fig. 3. Matrix representation of correlation coefficients between temperature variations on each pair of isobaric levels from 500 to $70 \mathrm{hPa}$.

\subsection{Calculation of integral indicators for anomalous variations of temperature time series}

We consider anomalous changes in amplitude and phase of temperature variations in the layers of atmosphere separated by tropopause as the main features characterizing temperature behavior of atmosphere before strong earthquakes (Fig. 4a).
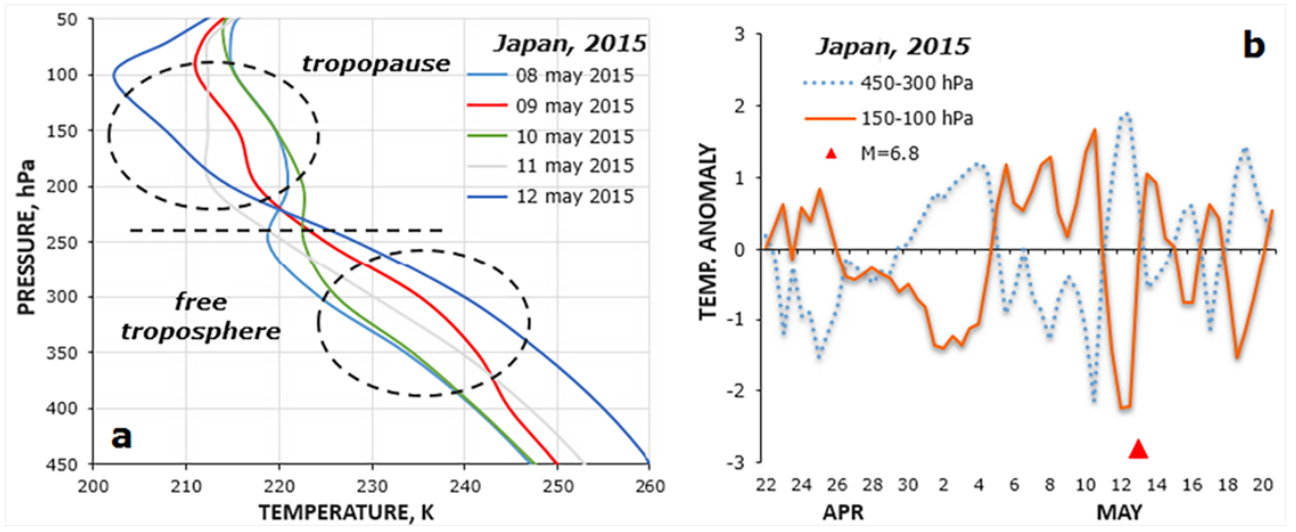

Fig. 4. Transformation of vertical temperature profiles (a) and time series of average values of temperature anomalies in 450-300 and 150-100 $\mathrm{hPa}$ layers (b) before the earthquake in Japan (marker indicates the moment of the earthquake $M=6.8$ on May 12, 2015).

This feature was taken as a basis for developing an algorithm for detecting anomalous temperature perturbations [2]. However, until recently the product of moving variations of temperature anomalies time series was calculated irrespective of the phase of temperature 
fluctuations. Therefore, to improve the reliability of pre-seismic anomalies detection $T(t)$, the procedure for calculating $D$ was corrected taking into account the correlation coefficient $(R)$ between temperature changes at the considered levels or in the atmospheric layers, designated by $D_{\text {CORR }}$. We calculated the integral index $D_{C O R R}$ in accordance with the following conditions: $D_{C O R R}=0$, if $R \geq 0$ and $D_{C O R R}=D \times|R|$, if $R<0$. That is, the $D_{C O R R}$ parameter resets to zero at positive values of the correlation coefficient and is adjusted depending on the value of $R$ with antiphase temperature changes (Fig. 4b). The inconsistency thus eliminated let us to diminish considerably although not to exclude the cases of greater than $2 \sigma$ "false" (or in-phase) anomalies.

\subsection{Analysis of anomalous values of $D$ integral parameter and seismic data}

The values of the product of temperature anomalies time series moving variations that are greater than one ( $D$ integral parameter) in free troposphere $\left(\Theta T_{F T}\right)$ and in tropopause $\left(\Theta T_{T P}\right)$ imply antiphase anomalies in short-term amplitude temperature variations in the considered layers of atmosphere, and, accordingly, high probability of seismic activity.

As experience has shown, the averaging of $\Theta T_{F T}$ and $\Theta T_{T P}$ for 2 or more isobaric levels is more preferable during calculations. This will provide additional "natural" data smoothing and selection of the most stable space-time variations.

\section{Results of detecting temperature anomalies}

Despite the amplitude and phase differences between temperature variations in the tropopause zone, determined by the seasonal conditions and geographic features of the considered earthquakes epicenters, implementation of the described algorithm made it possible to identify patterns (or features) common to all considered periods of seismic activity. A typical example of gradual temperature time series processing during foreshock activation and the main earthquake with magnitude $M=6.1$, which occurred on May 20, 2012 in Italy, is shown in Fig. 5.

The curves of temperature changes at different levels do not provide insight into consistency with seismicity by themselves (Fig. 5a) or they are not obvious, although the non-random nature of the effect is often seen quite well. At the same time, the results of processing temperature measurement data, obtained in accordance with the algorithm for individual isobaric atmospheric levels in March - June 2012, showed distinct temperature anomalies, which were observed several days before strong earthquakes. As it can be seen, zones with the highest values of $D$ parameter correlated with the main seismic impacts. The moments of anomalous temperature drop were quite short, and, which is the most important, they were directly connected to seismic shocks, preceded them (Fig. 5g). This feature of the atmospheric temperature behavior was tested for all selected cases of strong earthquakes in the Mediterranean region, thus confirming the results obtained previously [3]. 

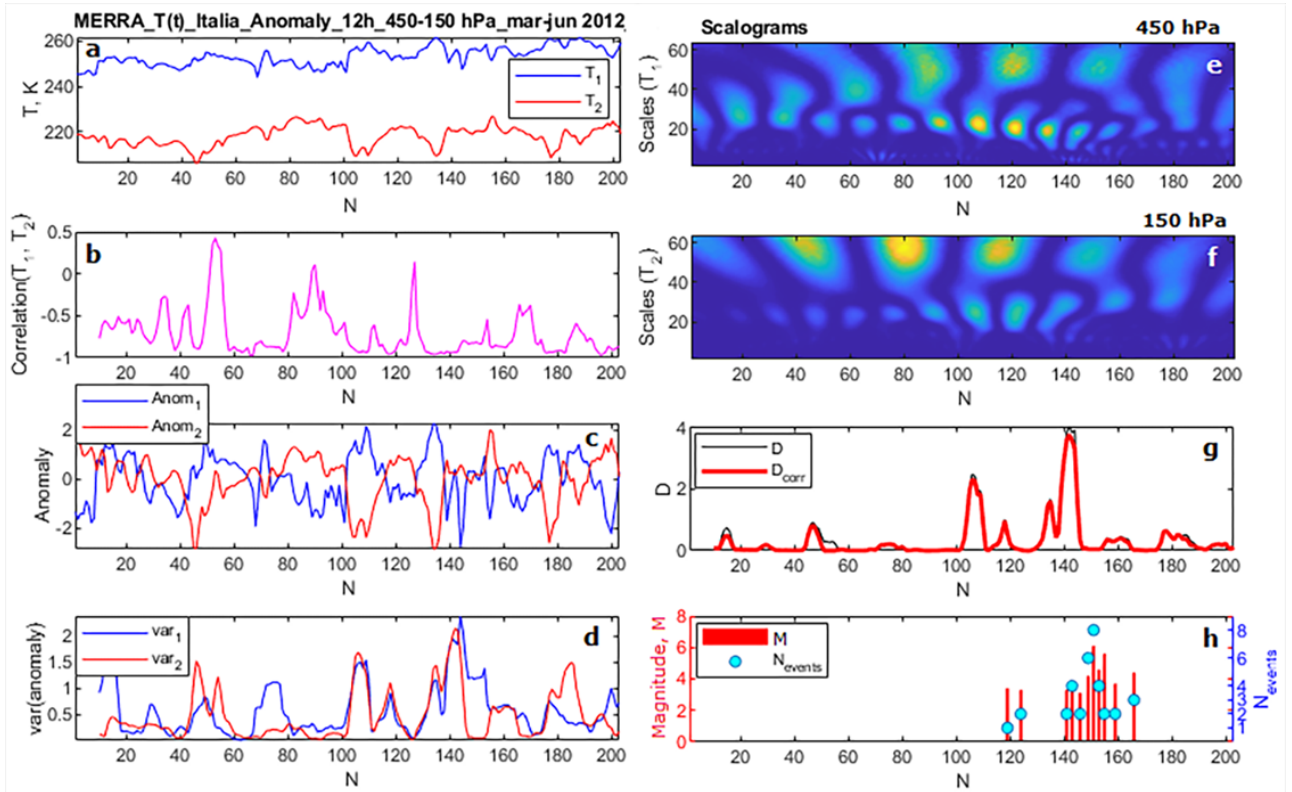

Fig. 5. Time series of temperature at levels of 450 and $150 \mathrm{hPa}$ (a), correlation coefficient $R$ (b), temperature anomalies (c) and their moving variations (d), wavelet coefficients spectra $W(a, b),(\mathrm{e}, \mathrm{f})$, $D$ parameter $(g)$, magnitude $M$ and number of earthquakes per day $N(\mathrm{~h})$ during the period from March to June $2012(M=6.1,05.20 .2012$, Italy).

\section{Conclusion}

The algorithm for processing data of satellite remote sensing, developed and used in this study, made it possible to allocate anomalies of short-term temperature variations in the tropopause during the foreshock activation before strong seismic events.

An analysis of satellite data showed that strong seismic events with magnitude of $M>6.0$ in the considered set that occurred in the Mediterranean region of Europe were preceded by an anomalous temperature change, which was observed about 1-10 days before the main shock and was followed by increase in the daily temperature range.

The results of remote sensing data processing revealed common features of anomalous temperature behavior synchronized with strong earthquakes in various regions of the world.

\section{References}

1. V.B. Kashkin. Atmospheric and Oceanic Optics, 26(10), 908-916. (2013)

2. V.B. Kashkin, A.A. Romanov, A.S. Grigoriev, A.A. Baskova. J. Sib. Fed. Univ. Eng. Technol. 5(2), 220-228. (2012)

3. L.G. Sverdlik, S.A. Imashev. J. Sib. Fed. Univ. Eng. Technol. 10(6), 783-793. (2017)

4. L.G. Sverdlik, S.A. Imashev. J. Sib. Fed. Univ. Eng. Technol. 11(8), 956-963. (2018)

5. M.R. Saradjian, M. Akhoondzadeh. Nat. Hazards Earth Syst. Sci. 11, 1109-1108. (2011)

6. World Meteorological Organization. WMO Bulletin. 4, 134. (1957)

7. V.M. Gadre, V.M. Dimri, E. Chandrasekhar. Boca Raton, FL: Taylor \& Francis Inc. 286 p. (2014) 